Hypotheses

\title{
New Opportunities for Fullerenes and for Graphene
}

\author{
Clive Delmonte \\ Open University, Clarendon Park Nottingham NG5 1AH, UK
}

Article history

Received: 24-02-2015

Revised: $15-05-2015$

Accepted: 18-09-2015

E-mail: clivedelmonte@gmail.com

\section{Summary}

Two fundamental features of modern chemistry are coordination chemistry (Gispert, 2008) and separately, the study of electrically conducting materials such as graphite. Latterly, of course, a single sheet of an electrical conductor such as graphene, with associated theory, has been the recent subject of a Nobel Prize (RSAS, 2010).

Aside of electrode driven oxidation and reduction, virtually all chemical and biochemical change takes place with no imposed electric potential. In this article is set out a means to impose electric potentials of choice so as to induce reactions not previously accessible, generally within unionized media.

The new class of substance chemically coordinates metal complexes or clusters within an electrically conducting framework upon which an electric potential is imposed. Whereas metals and their ions normally act at electric potentials within $\pm 3 \mathrm{v}$, an electrical potential can now be imposed, perhaps up to $\pm 15 \mathrm{v}$, or so, upon the metal or its ions which are immobilized and which are therefore constrained to act at an electric potential chosen by the researcher and novel to chemistry. As the framework of the polymer is not ionic but is covalent, it is reasonable to suggest that its stability will not be limited to that found in ionic compounds.

\section{Section a Synthesis of the Polymer}

Typically a notional intermediate, a monomer such as that shown in Diagram 1, can be polymerised. The empirical formula is $\mathrm{C}_{6} \mathrm{X}_{3}$ where $\mathrm{X}$, represented in Diagram 1, can be one or more of up to a further 15 elements or so, chosen from Groups 3A, 4A, 5A and 6A of the Periodic Table, or can be a combination of elements from these and sometimes other, Groups.

Typically $\mathrm{X}=\mathrm{N}, \mathrm{P}, \mathrm{As}$ or $\mathrm{Sb}, \mathrm{O}, \mathrm{S}, \mathrm{Se}$ and Te, or a selection of these ligand atoms which are known to form stable complexes with a wide range of metals, sometimes even as carbonyls or nitrosyls, or clusters and their ions.

The hexagonal ring is derived from benzene, known to form a perfect hexagon.

The monomer exemplified in Diagram 1, when $\mathrm{X}=$ hydroxyl $(\mathrm{OH})$ or amine $\left(\mathrm{NH}_{2}\right)$, can be polymerized to form a flat electrically conducting sheet on flat surfaces such as are found in graphene, Fullerenes and in graphite and can also be found in "white boron nitride", on mercury and in molybdenum sulfide. 


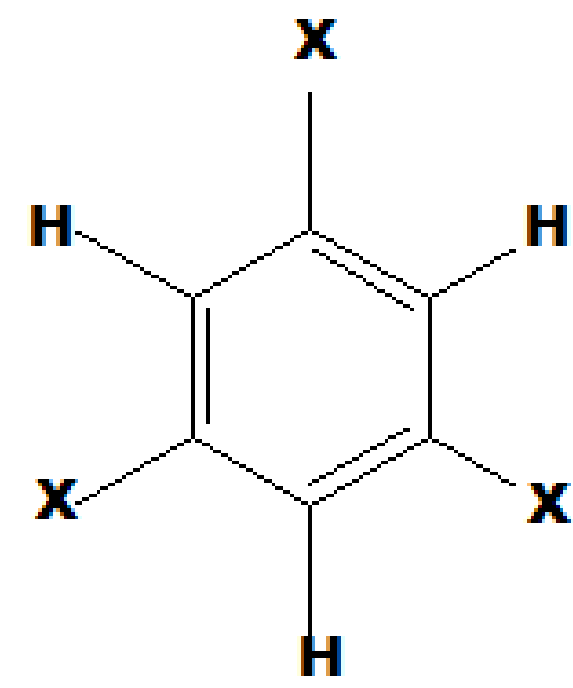

Diagram 1. A 1,3,5-substituted benzene

Though Fullerenes are synthesized in mixtures which generally contain $\mathrm{C}_{60}$ and $\mathrm{C}_{70}$ species, for example, it would not seem necessary to resolve such mixtures in order to successfully apply the processes outlined here.

Figure 1 represents an idealized scheme to use aniline under conditions of gentle oxidation to synthesize phenazene, which can be considered an intermediate in the formation of the high polymer in a twodimensional sheet.

The Periodic Table of Elements identifies some 60 metals and metalloids which could be coordinated in polymers such as those in Fig. 2 and 3 built from those based upon a monomer indicated in Diagram 1 and from Fig. 1.

This situation is therefore tantamount to the creation of a new chemistry for each of the ions, clusters and neutral compounds of some 50-60, mostly metallic, elements, when placed in a polymer such as that in Fig. 3 and itself lying upon a substrate such as a Fullerene or graphene.

In addition, antigens and proteins generally can be linked to the polymers by known chemical means.

During the synthesis and polymerization process to form the high polymer from the monomer in Diagram 1, cyclisation takes place as illustrated in Fig. 2 which illustrates the basic structural unit from which later, more complex polymers are assembled.

\section{The Synthesis of a Polymer with $N$ in the Ring Atoms}

A substrate is chosen from graphene nanoparticles (RSAS, 2010) or sonicated (nanoparticles) of graphite or Fullerene "soot" (Lieber and Chen, 1994) and a sample is stirred in a flask containing a medium of $50 \%$ aqueous ethanol at ambient temperatures. A known number of moles of phloroglucinol (1,3,5-trihydroxybenzene), in $50 \%$ aqueous ethanol is saturated with potassium hydroxide $(\mathrm{KOH})$, all at room temperature and added to the sample of substrate during stirring.<smiles>Nc1ccccc1</smiles>

Oxidation $-\mathrm{H}_{2} \mathrm{O}$<smiles>Nc1ccccc1</smiles><smiles>Nc1ccccc1</smiles>

\section{Oxidation}

$$
-\mathrm{H}_{2} \mathrm{O}
$$<smiles>N#[N+]c1ccccc1</smiles><smiles>N#Cc1ccccc1</smiles>

Oxidation

$-\mathrm{H}_{2} \mathrm{O}$<smiles>c1ccc2nc3ccccc3nc2c1</smiles>

\section{PHENAZENE}

Fig. 1. Gentle oxidation of aniline through to phenazene in an idealized sequence 


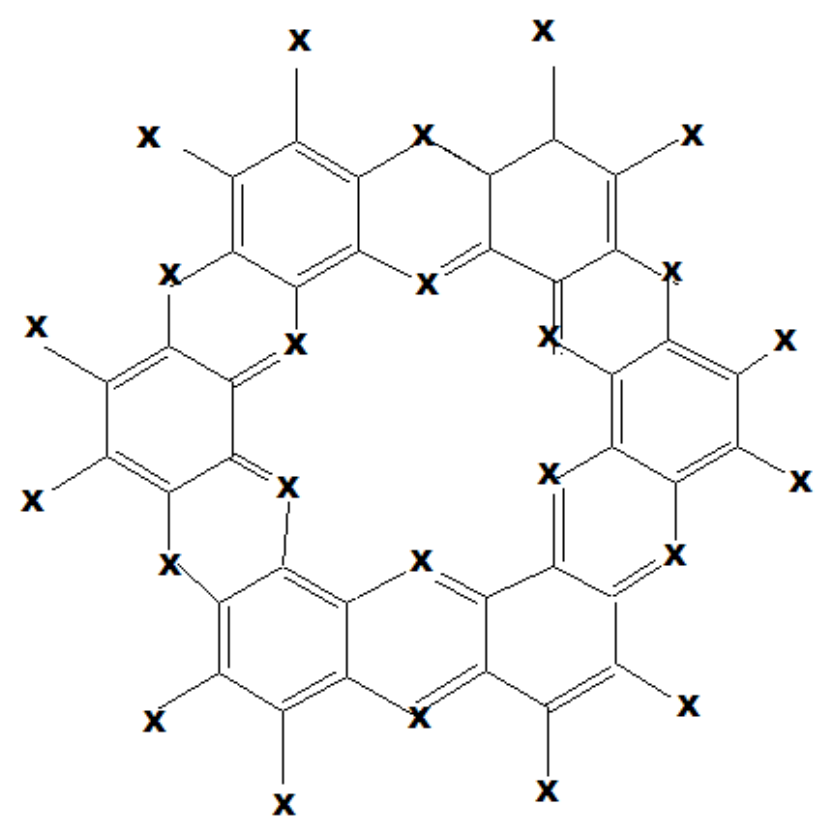

Fig. 2. Cyclophenazine when $\mathrm{X}=\mathrm{N}$

After a lapse of several minutes to allow alignment of the phloroglucinol ring with the substrate, a freshly prepared solution of hydroxylamine hydrochloride $\left(\mathrm{NH}_{2}(\mathrm{OH}) \cdot \mathrm{HCl}\right)$, itself dissolved in the minimum of $50 \%$ aqueous ethanol is added to the flask. The mole ratio of hydroxylamine: Phloroglucinol is maintained at least as 3:1.

The mixture is warmed rapidly to $80^{\circ} \mathrm{C}$ on a steam bath and clean air is passed through. An insoluble grey to black powder settles out, is washed in $50 \%$ aqueous ethanol and is freeze-dried. It takes a "twodimensional" structure as set out in Figure 3 due to its formation on the planar substrate. The polymer can be dubbed polycyclophenazene.

Figure 3 is evidently a fragment which is planar and has four large central "holes" in the piece illustrated, lending itself especially to the catalysis of gaseous reactions. Less obviously, the fragment benefits from an extended, distributed electron array which confers an electrical conductivity upon the polymer similar to that seen in graphite and graphene.

In addition, this synthesis does not require large specimens of graphene or carbon nanotubes, for example, but, acting as a catalyst, nanoparticles provide the greatest surface area per gram for catalytic action.

Again, when using $\mathrm{N}$, the central space can be considered as having a periphery consisting of bipyridyl and terpyridyl groups which are known to form especially stable complexes with $\mathrm{Fe}, \mathrm{Co}, \mathrm{Ni}, \mathrm{Cu}$ and $\mathrm{Zn}$ ions, for example.
From the standpoint of energetics, in such complexes, these ions would lie in a deep energy well. Thus a relatively large electric potential, perhaps up to $\pm 15 \mathrm{v}$ or more, can be applied to them without such ions or clusters being able to escape and without the covalent framework being disassembled. As "normal" ionic potentials are typically $\pm 3 \mathrm{v}$, researchers would find themselves in novel chemical domains.

\section{Synthesis of an Electrically Conducting Polymer Complex}

\section{Example $X=N$}

This is the most accessible example of the synthesis of an electrically conducting polymer starting from the monomer shown in Diagram 1.

After the synthesis from phloroglucinol and hydroxylamine described in some detail in an earlier paragraph, polymer forms as illustrated in Fig. 3.

The central chelating ring is large enough to accommodate metal complexes and part, or all of a metal cluster. To all of these an electric potential can be applied when contained in a metal pot after the polymer forms. The final hydrogen atoms have been removed during "polymerization" due to atmospheric oxidation.

An up-draft through a sintered disk under a fluidized bed in the metal pot would distribute evenly the imposed electric potential and temperature, both chosen by the researcher. The chemical reaction would proceed, for example, on the very high surface area of the Fullerene "soot" or graphene:

\section{D Substrates for Conducting Polymers}

As mentioned, an especially interesting substrate for polymers described here is Fullerene "soot" (Lieber and Chen, 1994). This is readily available in larger and larger amounts. The "soot" is an electrical conductor with some thermal stability and its structure has been studied by Xray diffraction (Scanlon and Ebert, 1993). A major component is spherical $\mathrm{C}_{60}$ which consists of an ordered array of 5- and 6-membered carbon rings and has some "aromatic" character.

The benzenoid structure of the polymers derived from Fig. 3 would be expected to adhere naturally via $\Pi: \Pi$ bonding to the aromatic regions, i.e., the delocalized electron character, of Fullerene soot, rather as layers of graphite might adhere to each other. This would obviate the need to devise explicit chemical bonding between the substrate and the polymer and thereby avoid disruption of the $\prod$ bonded electron layer.

In the case of graphene particles, both sides of the individual particles would acquire a catalytic capacity. 


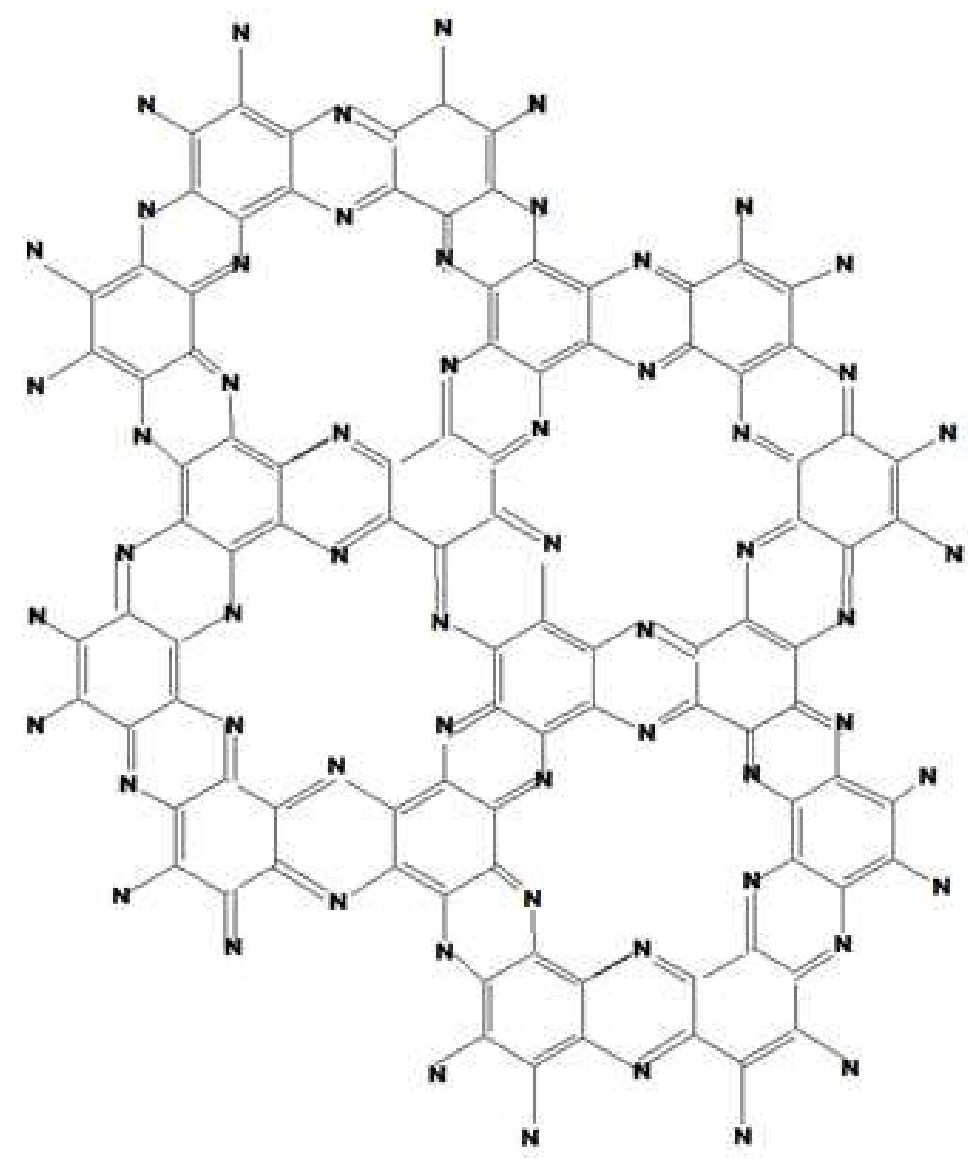

Fig. 3. Polycyclophenazene which is a Sketch of a Polymer Fragment which is Planar and has a delocalized $\prod$ electron layer which aligns itself on a similar layer on Fullerenes, graphene and graphite

\section{A Catalyst-Fullerene Soot Coated as a Catalyst}

A further feature of Fullerene soot is that many of its particles are nanoparticles (James, 2006) and typically can have diameters in the $1-5 \mu \mathrm{m}$ range. Together with its electrical conductivity, its chemical and thermal stability, its coating with a complex as in Fig. 3 and the very high surface area per gram of its many small particles, the soot would seem to be a potential catalyst support (Suib, 2013).

By varying the atom which replaces $\mathrm{X}$, using known methods, a wide range of entities can form potential catalysts.

The fluidized particles, when contained in an electrically conducting vessel and with a coordinated complex derived from Fig. 3, would distribute the imposed electric potential, the temperature and the chemical reaction uniformly.

The large central "holes" in Fig. 3 especially lend themselves and any coordinated complexes, to gas phase reactants and to coordination with large molecules and metal clusters.
Such catalysts might be exfoliated to recover the flat catalyst as a 2D monolayer, which is therefore a $2 \mathrm{D}$ crystal.

It is noteworthy that a perfectly flat surface of practically any size is accessible through use of a clean mercury layer as the fluid in a Langmuir-Blodgett trough. The mercury can be heated to promote polymerization and the polymer might be usable in situ since it lies on an electrical conductor.

The large specimens of polymer from the Langmuir-Blodgett trough might lend themselves to use as semi-conductors. These ideas have been brought together and elaborated in 61 Claims in UK patent application 1513830.8 .

\section{Some Applications of Conducting Complexes as in Figure 4}

Such complexes from Fig. 4, reacting under imposed high electric potentials, offer access to stable oxidation states and coordination numbers never before observed under ambient chemical potentials. 


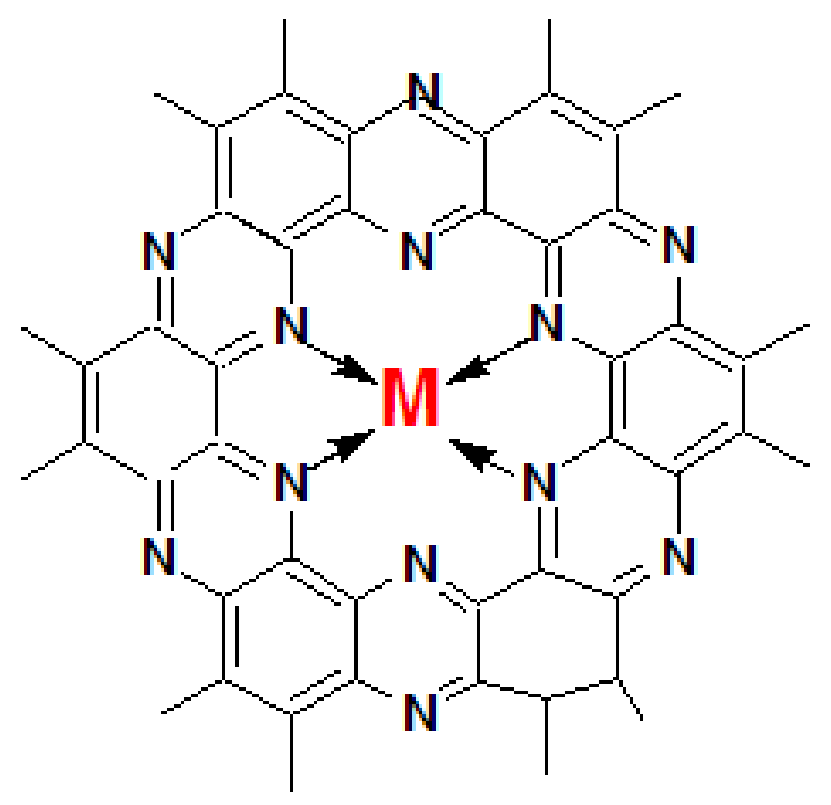

Fig. 4. An illustration of a complex with species $m$ coordinated with nitrogen

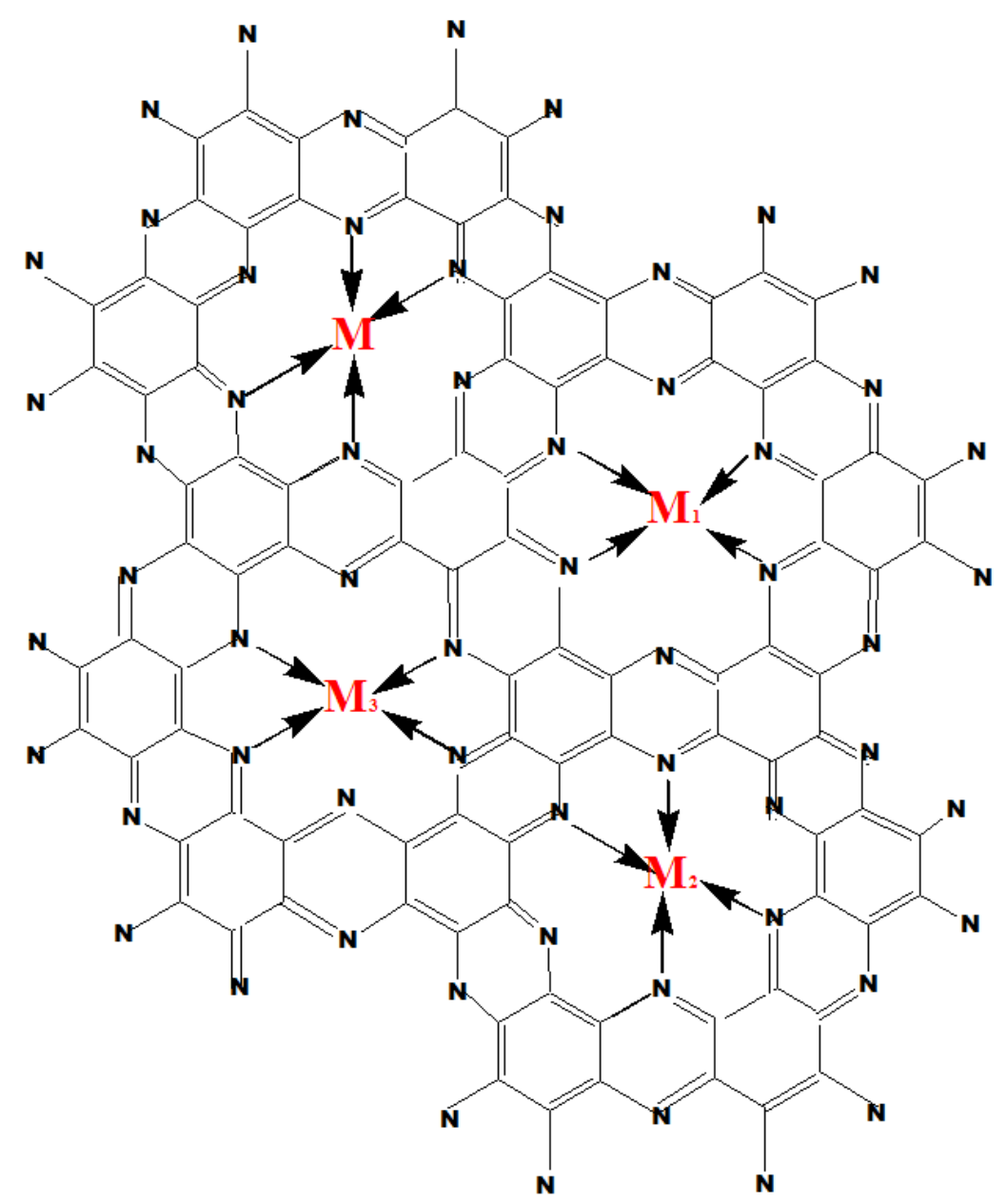

Fig. 5. An illustration of a complex with species $m$ through $m$ 
It is known that uranium has a normal upper valency of +6 , but with 9 outermost electrons, there is the possibility of higher valencies resulting from higher imposed electric potentials within polycyclophenazene. Again, thorium is of renewed interest in nuclear power generation by fission and has a normal upper valency of +4 . Its total of 10 outer electrons might imply that higher valencies could be accessible.

Many metals have a notional valency of zero, for example $\mathrm{Fe}$, Ni and $\mathrm{Mo}$, in their carbonyls and nitrosyls which might be further stabilized in polycyclophenazenes held at low electric potentials.

The Periodic Table allows the researcher to identify some 60 metals and metalloids which could be coordinated as $\mathrm{M}$ in polymers formed from those based upon the polymer indicated in Fig. 4, where an element as $\mathrm{X}$ is chosen from Group $3 \mathrm{~A}$ through to Group 6A atoms or a mixture of such atoms.

Thereafter, the researcher can select the electric potential to be applied to the catalyst.

Each of the ions, clusters and neutral compounds of some 50-60 elements, as $\mathrm{M}$, can coordinate to any of some 15 ligand elements as $\mathrm{X}$.

The high polymer in Fig. 5 is formed from that illustrated in Fig. 4. By known chemical means $M$ through M3 can be varied. This can be extended to antigens, short sequences of RNA and proteins as remarked earlier.

As stated earlier, $M$ can be chosen from a wide range of chemical moieties.

\section{Some Biomedical Applications}

The biological use of nanoparticles, both magnetized and otherwise, has been reported recently (El-Ansary and Faddah, 2010; Gruttner and Teller, 1999).

There would seem to be two principal categories of biochemical and biomedical applications for complexes with structures set out in Fig. 1 through 5, namely in vitro and in vivo. Several recent reviews of the biomedical applications of nanoparticles (Gruttner et al., 2001; Winfried et al., 2001; Kreyling et al., 2006) do not mention Fullerenes, Fullerene soot, applied electric potentials, or make explicit mention of working in vivo. These aspects seem novel and unexplored.

Though the application of even strong magnetic fields in vivo carries no known medical risk, exemplified by the use of MRI in scans of the human brain, the application of magnetic fields jointly with nanoparticles receives little attention in these reviews, perhaps because none of the probes is magnetic. Nevertheless, magnetic particles have been studied (Winfried et al., 2001; Kreyling et al., 2006).
In Figure 6, is set out an illustration of how the polymer in Fig. 5 might lie upon graphene or graphite, for example after the polymers of Fig. 4 and 5 have been loaded with particular moieties selected by the researcher.

However, Fullerene soot can be made magnetic with a suitable inclusion of iron compounds, for example and then external magnetic fields can be used to concentrate particles in vivo at chosen sites for therapeutic purposes, especially near the skin.

\section{In Vitro}

The smallest Fullerene particles would seem to be able to enter cells through their walls even if they carry new surface macrocycles from Fig. 5. These particles can have a range of markers attached to them, including biochemical sensors and antibodies, or antigens.

\section{In Vivo Applications}

In a more significant development, coated Fullerene particles might be deployed in vivo by injection, especially near the human brain and other parts of the central nervous system (Pfaltz and Drury, 2004; Krejcova et al., 2015). Targeted drug release might then be feasible to treat conditions near the body surface.

Then this new approach might lend itself to becoming part of the treatment of otherwise intractable conditions such as Alzheimer's disease, arthritis, dementia and rheumatism should it transpire that these conditions have a basis involving ionic species or species which can be given an electric charge, with or without a magnetic field or an intrinsic magnetic species in the complex.

In the Proceedings of the International Conference on Scientific and Clinical Applications of Magnetic Carriers (2001) is described the formation and use of magnetic nanoparticles (Hirn et al., 2011), for example.

Just as MRI scans are presently used for non-invasive mapping and monitoring and with no known "side effects", it may become possible to activate and monitor, such complexes through externally applied changes in electric potential running simultaneously with MRI scanning.

The advantage of using magnetic nanoparticles similar to the structure in Fig. 5 is that additional biomarkers and biosensors can be added to the particle surface to allow additional functions to be elaborated.

The use of nanoparticles composed of black iron oxide, $\mathrm{Fe}_{3} \mathrm{O}_{4}$, has been reviewed (Panagiotopoulos et al., 2015). A major advantage of a coating of polycyclophenazene is that it would help to reduce the tendency of the magnetic nanoparticles to aggregate together. The potential of such nanoparticles is an active area of research (Zhang et al., 2015; Kamble and Kolckar, 2014). 


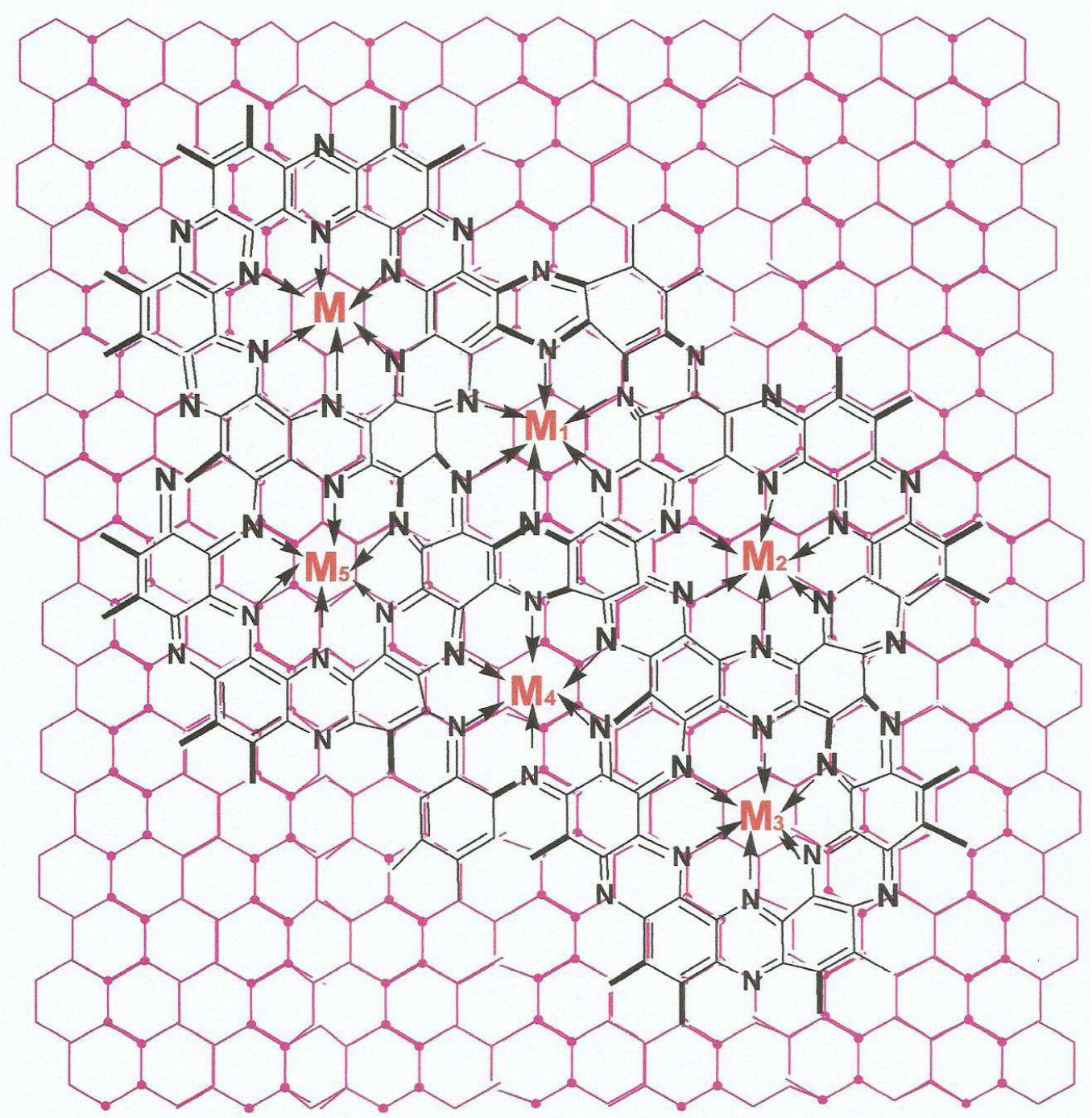

\section{M through M5 are variously metal atoms, ions or part of a metal cluster which can coordinate to nitrogen (in this example) and where M to M5 may be the same or different from each other, e.g., typically $\mathrm{Ti}, \mathrm{V}, \mathrm{Cr}, \mathrm{Mn}, \mathrm{Fe}, \mathrm{Co}, \mathrm{Ni}, \mathrm{Cu}$ or $\mathrm{Zn}$}

Fig. 6. A fragment of a sheet of polycyclo-phenazene in a complex with species $\mathrm{m}$ and lying upon graphene

\section{Conclusion}

Some superficially simple chemical reactions offer prospects of being catalyzed using complexes from Fig. 6 which individually and collectively, have the potential to transform and improve human prospects.
The catalytic properties of a new class of electrically conducting macrocyclic catalysts which can coordinate a wide range of chemical moieties at electric potentials new to Chemistry may help to inaugurate a fresh era of nano-technological change. 


\section{Ethics}

This article is original and contains unpublished material. The corresponding author confirms that all of the other authors have read and approved the manuscript and no ethical issues involved.

\section{References}

Gispert, J.R., 2008. Coordination Chemistry. 1st Edn., Wiley, Weinheim, ISBN-10: 352731802X, pp: 600.

RSAS, 2010. Royal Swedish academy of sciences. Graphene.

Lieber, C. and C. Chen, 1994. Preparation of fullerenes and fullerene-based materials. Solid State Phys., 48: 109-148.

Scanlon, J. and L. Ebert, 1993. X-ray diffraction study of fullerene soot. J. Phys. Chem., 97: 7138-7140.

James, A.L., 2006. Kinetics, catalysis and mechanism of methane steam reforming. MSc Thesis, Department of Chemical Engineering, Worcester Polytechnic Institute, UK.

Suib, S.L., 2013. New and Future Developments in Catalysis: Catalysis by Nanoparticles. 1st Edn., Newnes, Amsterdam, ISBN-10: 0444538755, pp: 512.

El-Ansary, A. and L.M. Faddah, 2010. Nanoparticles as biochemical sensors. Nanotechnol. Sci. Appl., 3: 65-76. DOI: 10.2147/NSA.S8199

Gruttner, C. and J. Teller, 1999. New types of silicafortified magnetic nanoparticles as tools for molecular biology applications. J. Magnetism Magnetic Mater., 194: 8-15.

DOI: $10.1016 / \mathrm{S} 0304-8853(98) 00561-7$

Gruttner, C., S. Rudershousen and J. Teller, 2001. Improved properties of magnetic particles by combination of different polymer materials as particle matrix. J. Magnetism Magnetic Mater., 225: 1-7. DOI: 10.1016/S0304-8853(00)01220-8

Winfried, M., G. Scheuch, K. Sommerer and J. Heyder, 2001. Preparation of spherical monodisperse ferrimagnetic iron-oxide microparticles between 1 and $5 \mu \mathrm{m}$ diameter. J. Magnetism Magnetic Mater., 225: 8-16. DOI: 10.1016/S0304-8853(00)01221-X
Kreyling, W.G., M. Semmler-Behnke and W. Möller, 2006. Health implications of nanoparticles. J. Nanoparticle Res., 8: 543-562. DOI: 10.1007/s11051-005-9068-Z

Pfaltz, A. and W. Drury, 2004. Design of chiral ligands for asymmetric catalysis: From C2-symmetric P,Pand N,N-ligands to sterically and electronically nonsymmetrical P,N-ligands. Proc. Nat. Acad. Sci., 101: 5723-5726. PMID: 15069193

Krejcova, L., P. Michalek, M.M. Rodrigo, Z. Heger and S. Krizkova et al., 2015. Nanoscale virus biosensors: State of the art. Dovepress, 2015: 47-66. DOI: $10.2147 /$ NDD.S56771

Hirn, S., M. Semmler-Behnke, C. Schleh, A. Wenk and J. Lipka et al., 2011. Particle size-dependent and surface charge-dependent biodistribution of gold nanoparticles after intravenous administration. Eur. J. Pharm. Biopharm., 77: 407-416. DOI: $10.1016 /$ j.ejpb.2010.12.029

Panagiotopoulos, N., R.L Duschka, M. Ahlborg, G. Bringout and C. Debbeler et al., 2015. Magnetic particle imaging: Current developments and future directions. Int. J. Nanomedicine, 10: 3097-3114. DOI: $10.2147 /$ IJN.S70488

Zhang, W., L. Qiao, X. Wang, R. Senthilkumar and F. Wang et al., 2015. Inducing cell cycle arrest and apoptosis by dimercaptosuccinic acid modified $\mathrm{Fe}_{3} \mathrm{O}_{4}$ magnetic nanoparticles combined with nontoxic concentration of bortezomib and gambogic acid in RPMI-8226 cells. Int. J. Nanomedicine, 10: 3315-3328. DOI: 10.2147/IJN.S80795

Kamble, V. and S. Kolckar, 2014. Analysis of rheological properties of MR fluid based on variation in concentration of iron particles. Am. J. Nanotechnol., 5: 12-16. 